\section{Perfil epidemiológico de neoplasias epiteliales de glándulas salivales}

\author{
ANDRÉS CAMPOLO GONZÁLEZ , HERNÁN RAMÍREZ SKINNER ${ }^{1}$ \\ ALEX VARGAS DÍAZ ${ }^{1}$, AUGUSTO LEÓN RAMÍREZ ${ }^{1}$, \\ IGNACIO GOÑI ESPILDORA ${ }^{1}$, ANTONIETA SOLAR GONZÁLEZ ${ }^{2}$
}

\section{Epithelial tumors of salivary glands. Review of 286 pathology reports}

Background: Epithelial tumors of the salivary glands, including benign tumors and aggressive malignancies with different prognoses, are uncommon. Aim: To describe the frequency and distribution of salivary gland tumors according to age, gender and anatomical location. Material and Methods: Review of pathological reports of salivary gland tumors of a Pathology laboratory at a clinical hospital from 2006 to 2016. Results: Five hundred ninety salivary gland biopsies were reviewed. Of these, 286 (49\%) were primary epithelial tumors of the salivary glands. Two hundred thirty (80\%) were benign and 56 (20\%) were malignant tumors. Regarding location, 274 (96\%) were in the major salivary glands, and $12(4 \%)$ in the minor salivary glands. The most common histological types were pleomorphic adenoma for benign tumors in 172 cases, followed by papillary cystadenoma lymphomatosum in 33 cases. Mucoepidermoid carcinoma was the most common malignant tumor in 14 cases. Conclusions: These results are similar to reports from abroad, however more studies are necessary to be able to establish a more representative and updated analysis.

(Rev Med Chile 2018; 146: 1159-1166)

Key words: Neoplasms; Pathology; Salivary Glands; Surgery, Oral.

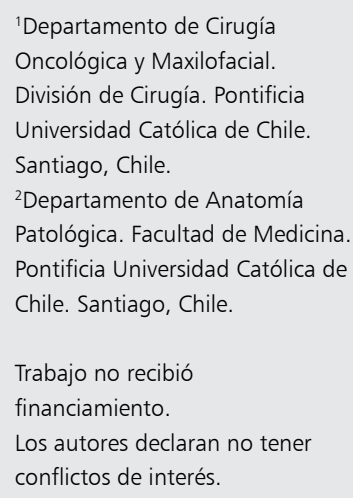

Recibido el 4 de abril de 2018, aceptado el 30 de octubre de 2018.

Correspondencia a:

Andrés Campolo González Dirección: Marcoleta \#350,

Santiago, Chile.

andres.campolo@gmail.com

\section{I} as glándulas salivales se distribuyen ampliamente en la región de cabeza y cuello. Las glándulas salivales mayores corresponden a la glándula parótida, submandibular y sublingual, mientras que las glándulas salivales menores son muchas distribuidas en múltiples sitios como la mucosa labial, mucosa yugal, paladar, lengua, piso de boca y orofaringe ${ }^{1}$.

Las neoplasias de glándulas salivales son poco frecuentes. De acuerdo a la literatura corresponden a 3-6\% de los tumores de cabeza y cuello, y a $0,6 \%$ de todas las neoplasias. Tienen una incidencia global estimada en $0,4-13,5$ por cada 100.000 personas anualmente y éstas incluyen un grupo histopatológico heterogéneo que va desde neoplasias benignas hasta neoplasias malignas muy agresivas y de mal pronóstico ${ }^{1-4}$.

La distribución del sitio, edad y sexo de los pacientes afectados por los distintos tipos de neoplasias de glándulas salivales varían de acuerdo a la raza, el tipo histológico y la ubicación geográfica $^{5}$. Estudios realizados por Tian et al y por Jaber et al, dónde se compararon sus resultados con otras series demostraron que la incidencia de estas neoplasias varían de acuerdo al área geográfica ${ }^{2,5}$.

En la actualidad existe escasa evidencia actualizada respecto a la distribución de este tipo de patologías en Chile $e^{4,6,7}$.

El objetivo de este estudio es describir la frecuencia relativa y distribución de las neoplasias de glándulas salivales de acuerdo a edad, género y ubicación anatómica, de los pacientes evaluados durante los últimos 10 años en el Laboratorio de Anatomía Patológica del Hospital Clínico de la Pontificia Universidad Católica de Chile. 


\section{Materiales y Método}

Estudio epidemiológico transversal descriptivo realizado en el Laboratorio de Anatomía Patológica del Hospital Clínico de la Pontificia Universidad Católica de Chile.

Se recolectaron datos a través de la revisión de informes anatomo-patológicos de glándulas salivales pertenecientes a pacientes evaluados en el período comprendido entre mayo de 2006 a mayo de 2016.

Todos los casos de neoplasias epiteliales primarias de glándulas salivales fueron revisados, registrados y reclasificados (si es necesario) de acuerdo a los Criterios de Clasificación para los Tumores de Cabeza y Cuello de la Organización Mundial de la Salud (OMS) del año 2005 y su actualización en la $4^{\text {a }}$ edición de la OMS (2017) ${ }^{8}$.

La información recolectada incluye: fecha de admisión, edad, género, localización, diagnóstico, presentación histopatológica (variedad, grado histológico). No se registró ningún dato personal en esta investigación. Los datos fueron recolectados a través del software Epi Info ${ }^{\mathrm{TM}}$ software (versión 7.0, CDC, Atlanta, USA) y se utilizó estadística descriptiva para presentar los resultados.

La realización de este trabajo fue aprobado por el Comité de ética científico de la Facultad de Medicina de la Pontificia Universidad Católica de Chile.

\section{Resultados}

En el período comprendido entre mayo de 2006 a mayo de 2016, se registraron un total de 590 biopsias de glándulas salivales, de las cuales 286 correspondieron a diagnósticos de neoplasias epiteliales primarias de glándulas salivales, representando $48,5 \%$ del total. Las otras 304 biopsias correspondieron a sialadenitis (19\%), Sjøgren(15,3\%), mucoceles (2\%), glándulas salivales normales $(1,5 \%)$ y otras lesiones $(13,7 \%)$.

\section{Tipos histológicos}

Los 286 casos de neoplasias epiteliales primarias de las glándulas salivales no fueron casos repetidos, se encontraron en 286 pacientes diferentes. De éstos, $230(80,4 \%)$ correspondieron a neoplasias benignas, mientras que 56 registros $(19,5 \%)$ fueron neoplasias malignas. La propor- ción tumor benigno con tumor maligno fue de $4,1: 1$. Las distribuciones por tipo histológico y localización de las neoplasias epiteliales benignas y malignas de glándulas salivales se muestran en las Tablas 1 y 2, respectivamente.

La neoplasia benigna más frecuente fue el adenoma pleomorfo representando 74,7\% (172/230 casos), seguido por el cistadenoma papilar linfomatoso con 14,3\% (33/230). Adenoma de células basales y mioepitelioma siguen en frecuencia representando 3,9\% (9/230) y 3,0\% (7/230) respectivamente. No se registraron casos de adenoma sebáceo, papiloma ductal invertido, papiloma intraductal, sialoadenoma papilífero y cistoadenoma (Tabla 1).

Dentro del grupo de neoplasias malignas de glándulas salivales, el diagnóstico más frecuente correspondió a carcinoma mucoepidermoide con $25 \%$ de los casos (14/56), seguido por el carcinoma de células acinares y carcinoma adenoideo quístico los cuales tienen 9 registros cada uno, correspondiente a $16,0 \%$ del total. El tercer tipo histológico más frecuente correspondió al carcinoma ex-adenoma pleomorfo con 12,5\% (7/56).

En los casos de carcinoma ex adenoma pleomórfico, encontramos asociación con un componente de carcinoma mioepitelial en 4 casos, carcinoma de conducto salival en 2 casos y adenocarcinoma de sitio no especificado en 1 caso.

No se registraron casos de carcinoma de células claras, adenocarcinoma de células basales, linfoadenocarcinoma sebáceo, cistoadenocarcinoma, cistoadenocarcinoma cribiforme de bajo grado, adenocarcinoma mucinoso, carcinosarcoma, adenoma pleomorfo metastatizante, carcinoma de células pequeñas ni sialoblastoma (Tabla 2).

\section{Localización}

De todas las lesiones registradas, 95,8\% (274/286) correspondieron a neoplasias de glándulas salivales mayores, mientras que solo $4,2 \%$ (12/286) en las glándulas salivales menores. De los tres pares de glándulas salivales mayores, la más afectada fue la glándula parótida, representando $82,8 \%$ de todas las neoplasias (237/286) seguido por la glándula submandibular de la cual se registraron 37 casos $(12,9 \%)$. No hubo casos informados en glándula sublingual. Respecto a la proporción de lesiones benignas con malignas para cada glándula salival, tanto en glándula parótida como en submandibular se vio una mayor 


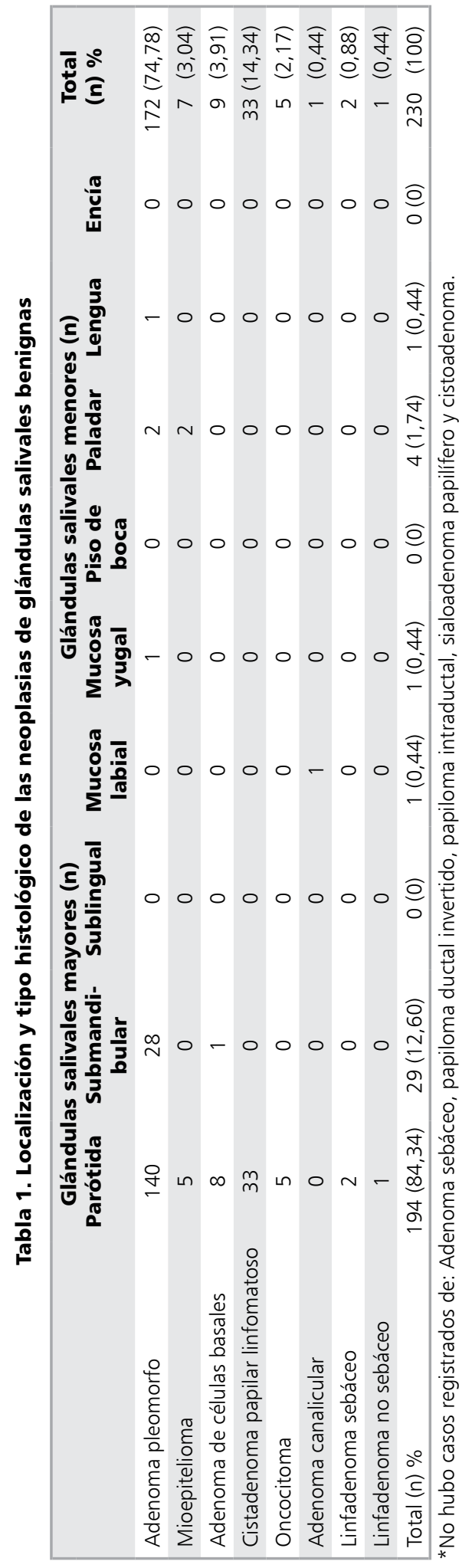

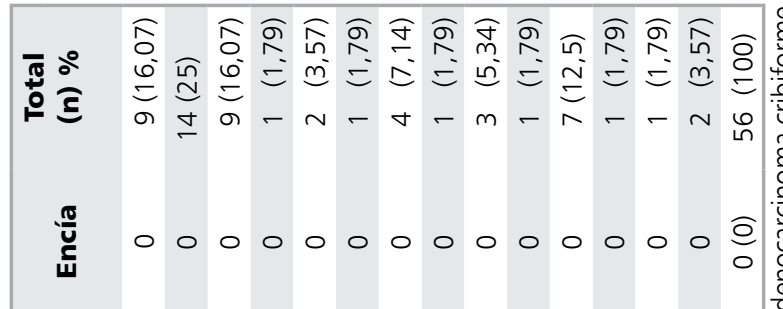

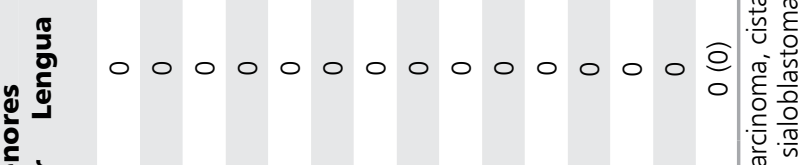

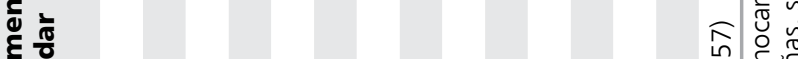

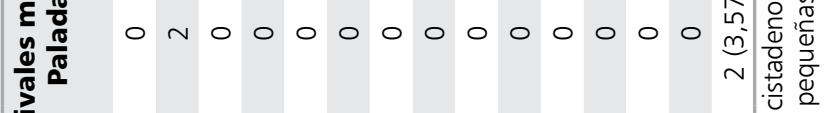

สำ ฮ

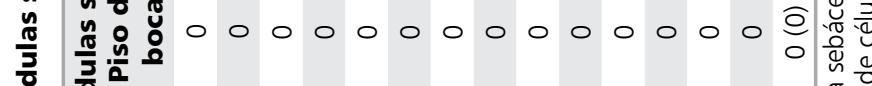

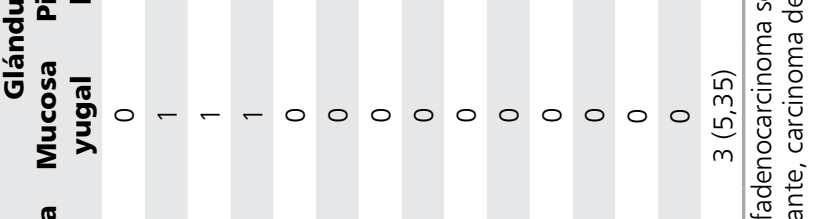

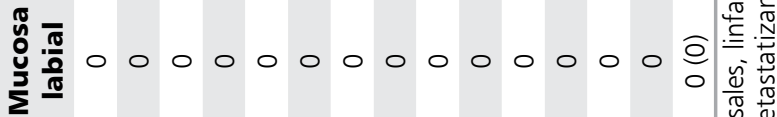

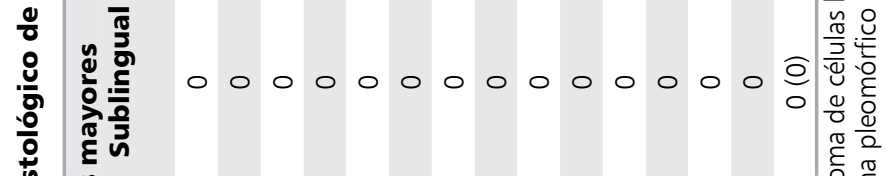

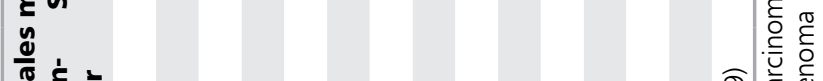

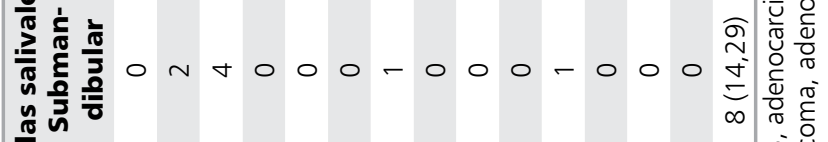

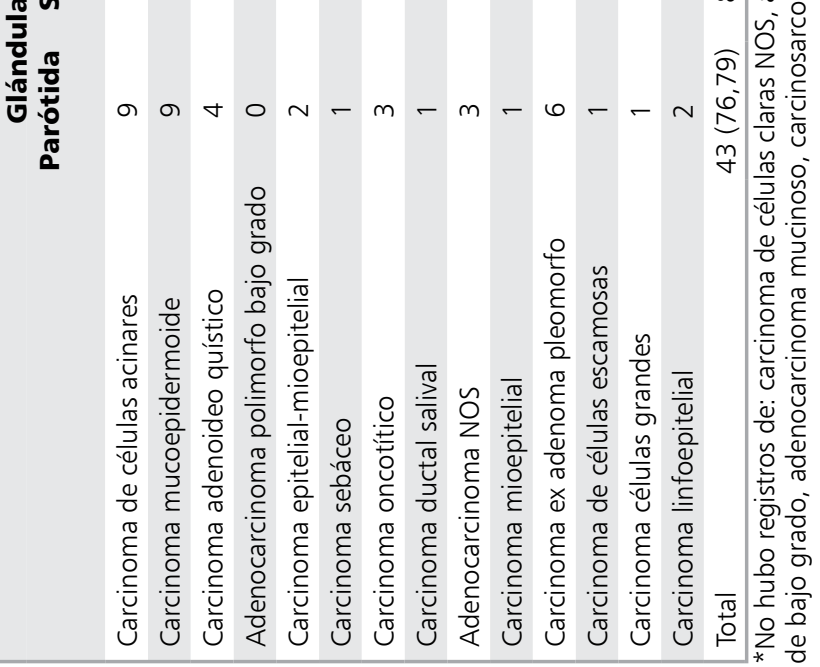


prevalencia de neoplasias salivales benignas; 4,5:1 y 3,6:1 respectivamente (Figura 1).

Dentro del grupo de glándulas salivales menores, la región más afectada correspondió al paladar con $2,0 \%(6 / 286)$, seguido por mucosa yugal con $1,3 \%(4 / 286)$. Solo se observó 1 caso de neoplasia en labio y lengua, representando ambos $0,3 \%$. No se encontraron casos de glándulas salivales en piso de boca o mucosa gingival.

En general, la proporción de neoplasias benignas con malignas fue a favor de las primeras, con excepción de la mucosa yugal, en donde la proporción fue de 1:3. Para mucosa labial, paladar y lengua, las proporciones fueron de 1:0, 2:1 y 1:0 respectivamente (Figura 1).

Los tipos histológicos más frecuentes en la parótida fueron el adenoma pleomorfo (140/237), cistadenoma papilar linfomatoso (33/237), carcinoma de células acinares (9/237), y carcinoma mucoepidermoide (9/237). De la glándula submandibular se registraron principalmente casos de adenoma pleomorfo (28/37), seguido por carcinoma adenoideo quístico $(4 / 37)$ y carcinoma mucoepidermoide (2/37) (Tablas 1 y 2 ).

En glándulas salivales menores los tipos más frecuentes fueron adenoma pleomorfo $(4 / 12)$, luego carcinoma mucoepidermoide (3/12), mioepitelioma (2/12), carcinoma adenoideo quístico (1/12), adenocarcinoma polimorfo de bajo grado (1/12), y adenoma canalicular (1/12).

\section{Edad y género}

De los 286 casos registrados, $100(34,9 \%)$ correspondieron pacientes de género masculino y $186(65,0 \%)$ a pacientes de género femenino, con una proporción hombre-mujer 1:1,86. Dentro de las neoplasias salivales benignas se registró una proporción similar, pacientes de sexo masculino fueron 78 y sexo femenino 152 con una proporción hombre-mujer de 1:1,95. Por último en el grupo de las neoplasias malignas, los pacientes de sexo masculino fueron 22 , mientras que las de sexo femenino 34, con una proporción hombre-mujer de 1:1,54 (Tabla 3).

Dentro de los distintos tipos histológicos presentes en el estudio, también pudimos encontrar variaciones, siendo más marcada la diferencia por géneros en el adenoma pleomorfo y en el carcinoma mucoepidermoide. Las proporciones hombre-mujer de los tipos histológicos recién

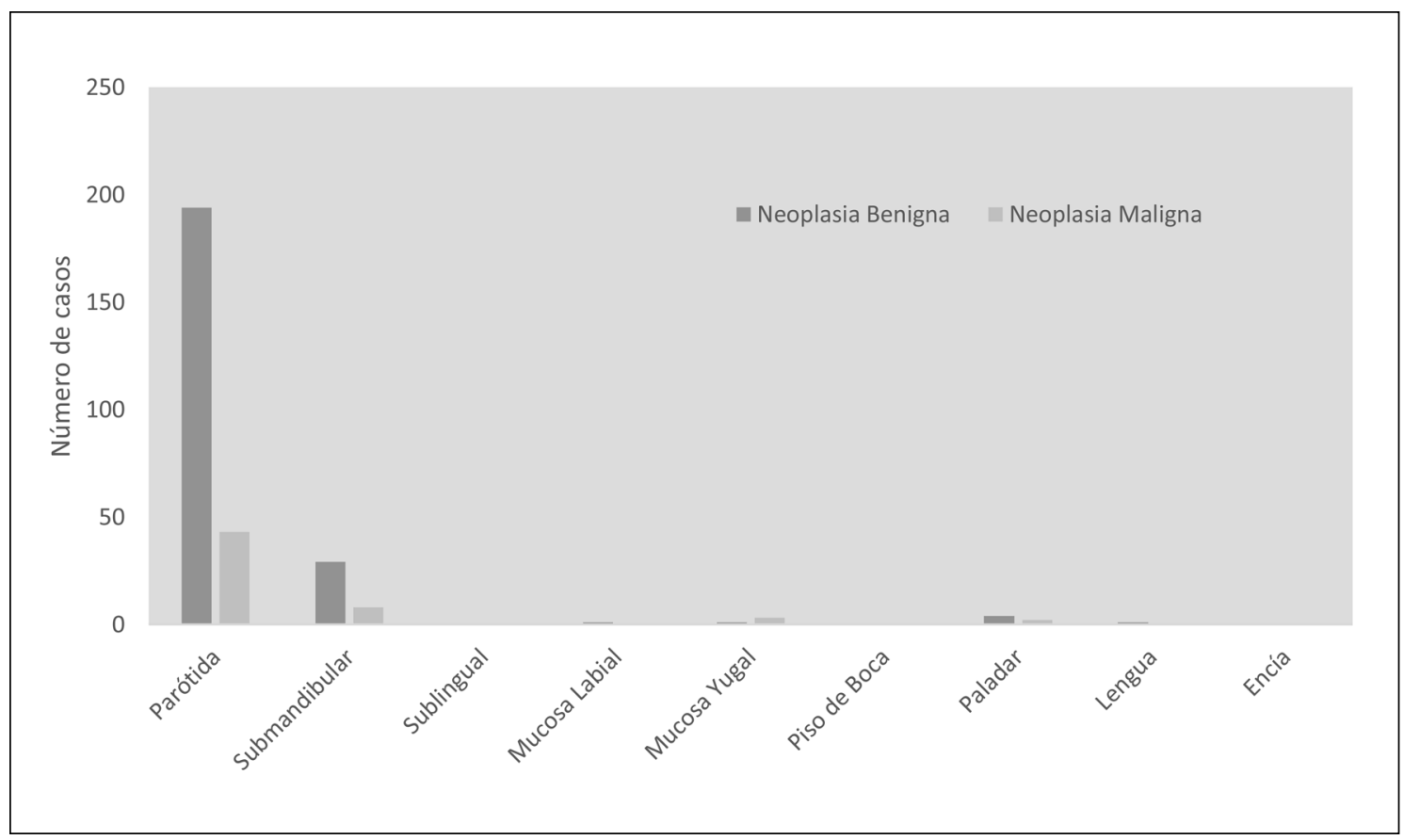

Figura 1. Distribución de la localización anatómica de las neoplasias de glándulas salivales. 
Tabla 3. Distribución por género de las neoplasias de glándulas salivales

\begin{tabular}{|c|c|c|c|}
\hline & Masculino (n) & Femenino (n) & Hombre:Mujer \\
\hline \multicolumn{4}{|l|}{ Neoplasias benignas } \\
\hline Adenoma pleomorfo & 51 & 121 & $1: 2,37$ \\
\hline Mioepitelioma & 4 & 3 & $1,3: 1$ \\
\hline Adenoma de células basales & 3 & 6 & $1: 2$ \\
\hline Cistadenoma papilar linfomatoso & 17 & 16 & $1,1: 1$ \\
\hline Oncocitoma & 2 & 3 & $1: 1,5$ \\
\hline Adenoma canalicular & 0 & 1 & - \\
\hline Linfadenoma sebáceo & 0 & 2 & - \\
\hline Linfadenoma no sebáceo & 1 & 0 & - \\
\hline Total & 78 & 152 & $1: 1,9$ \\
\hline \multicolumn{4}{|l|}{ Neoplasias malignas } \\
\hline Carcinoma de células acinares & 5 & 4 & 1,3:1 \\
\hline Carcinoma mucoepidermoide & 3 & 11 & $1: 3,7$ \\
\hline Carcinoma adenoideo quístico & 4 & 5 & 1,3:1 \\
\hline Adenocarcinoma polimorfo bajo grado & 0 & 1 & - \\
\hline Carcinoma epitelial-mioepitelial & 1 & 1 & $1: 1$ \\
\hline Carcinoma sebáceo & 0 & 1 & - \\
\hline Carcinoma oncotítico & 2 & 2 & $1: 1$ \\
\hline Carcinoma ductal salival & 1 & 0 & - \\
\hline Adenocarcinoma NOS & 0 & 3 & - \\
\hline Carcinoma mioepitelial & 0 & 1 & - \\
\hline Carcinoma ex adenoma pleomorfo & 4 & 3 & $1,3: 1$ \\
\hline Carcinoma de células escamosas & 1 & 0 & - \\
\hline Carcinoma células grandes & 1 & 0 & - \\
\hline Carcinoma linfoepitelial & 0 & 2 & - \\
\hline Total & 22 & 34 & $1: 1,5$ \\
\hline
\end{tabular}

nombrados fueron de 1:2,4 y 1:3,7 respectivamente. Por otro lado, pudimos observar que el cistadenoma papilar linfomatoso y el carcinoma de células acinares presentaban una frecuencia levemente mayor en pacientes de sexo masculino, con proporciones hombre-mujer de 1,1:1 y 1,25:1 (Tabla 3).

Respecto a la distribución por edad, pudimos observar que la mayor frecuencia se presentó en la sexta década de vida (51 a 60 años) con 61/286 casos (21,3\%). Dentro de las neoplasias benignas, la sexta década fue la más frecuente representando $22,1 \%(51 / 230)$, mientras que para las neoplasias malignas, el peak estuvo en el grupo de la cuarta década (31 a 40 años), siendo 19,6\% (11/56). (Figura 2) La edad promedio total correspondió a 48 años, mientras que la edad promedio para las neoplasias benignas y malignas fueron $47 \mathrm{y}$ 52 respectivamente. En el grupo etario menor a 20 años se registraron solo 16 casos, los cuales representan 5,6\% del total, y de estos 14 fueron tipos histológicos benignos y 2 malignos. 


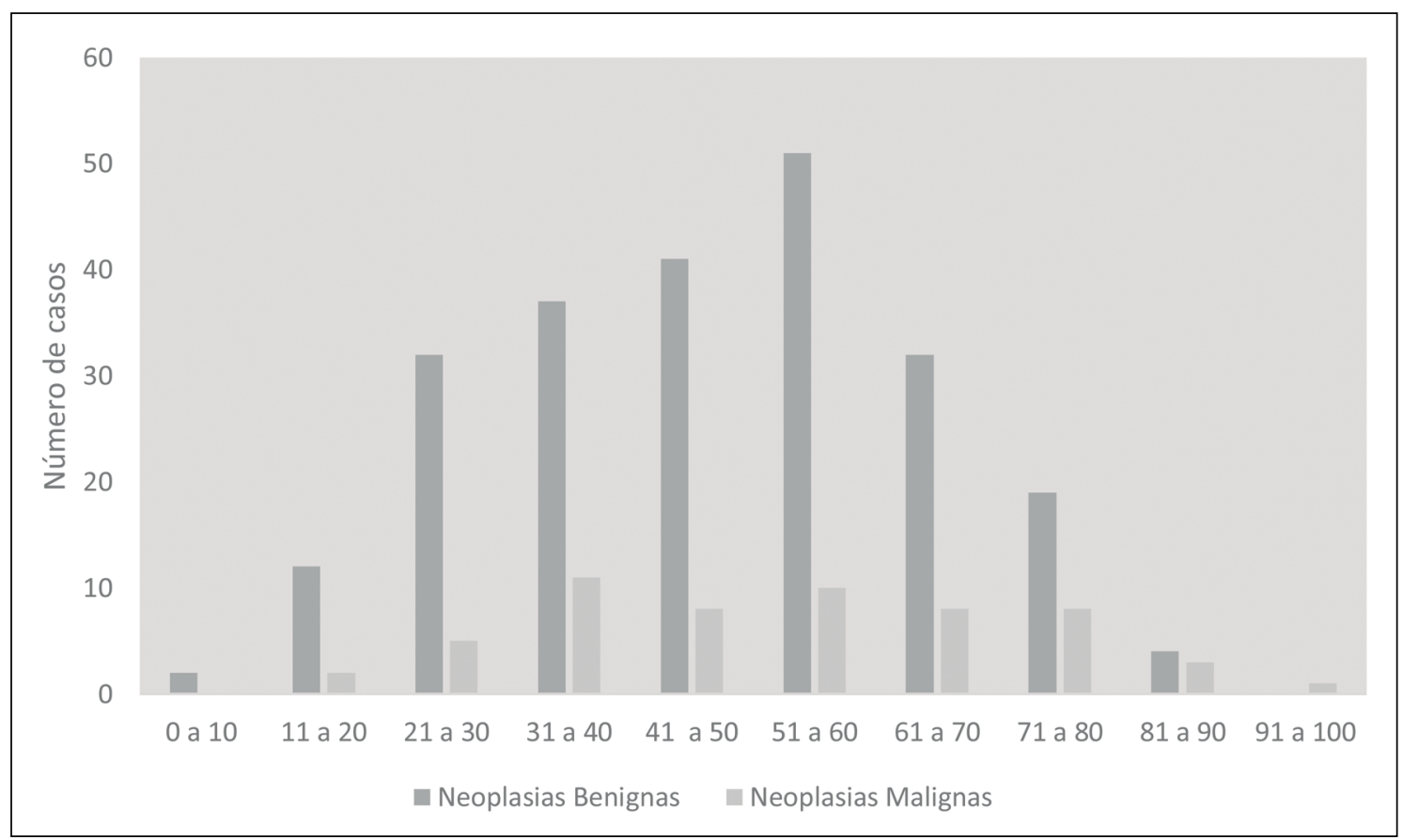

Figura 2. Distribución por grupo etario de neoplasias de glándulas salivales.

\section{Discusión}

A nivel nacional existe escasa literatura actualizada respecto a la epidemiología de neoplasias epiteliales de glándulas salivales. Desde el año 2005 existe una nueva clasificación propuesta por la OMS, dando una mayor precisión a los distintos tipos histopatológicos de las neoplasias. Para efectos de este trabajo no adquiere mucha relevancia puesto que todos los diagnósticos anatomo-patológicos revisados son desde el año 2006, sin embargo, y para evitar confusiones, los cortes histológicos fueron revisados y reclasificados por el mismo especialista para confirmar los resultados.

Las neoplasias benignas fueron las más frecuentes, al igual que en todos los estudios evaluados, registrándose por sobre $60 \%$ de los casos. Esto sugiere que las neoplasias benignas en glándulas salivales serían más frecuentes a nivel mundial.

Por otro lado, las neoplasias malignas correspondieron a $19,5 \%$ de los casos registrados, resultado similar a series de Brasil ${ }^{10,14}$, pero que difieren a otros estudios a nivel mundial donde se reportan frecuencias entre 32-38\% (Tabla 4). Lo anterior podría sugerir una variación geográfica en la frecuencia de las neoplasias malignas de glándulas salivales, así como variaciones locales en las modalidades de estudio diagnóstico y criterios de indicación quirúrgica, reduciendo el número de paciente operados por neoplasias benignas y afectando directamente a las frecuencias reportadas.

El adenoma pleomorfo fue el tipo histológico más común, representando $60,1 \%$ de todas las neoplasias de glándulas salivales y 74,7\% de las neoplasias benignas. Estos resultados son compatibles con la literatura en donde el predominio de este tipo histológico se reporta en todas las series analizadas (Tabla 4). El segundo tipo más frecuente en el presente estudio correspondió al cistadenoma papilar linfomatoso, con 11,5\% de todas las neoplasias salivales y $14,3 \%$ de las neoplasias benignas.

Estos resultados se corresponden con la evidencia nacional e internacional ${ }^{2,6,9,10}$, sin embargo, existen estudios que han reportado al cistadenoma papilar linfomatoso como la tercera neoplasia más frecuente después del carcinoma mucoepidermoide o el carcinoma adenoideo quístico ${ }^{4,11,12}$.

El tercer tipo histológico más frecuente en esta serie, fue el carcinoma mucoepidermoide, presen- 
Tabla 4. Comparación en distribución de neoplasias de glándulas salivales con otros países

\begin{tabular}{|c|c|c|c|c|c|c|c|c|c|}
\hline País & $\begin{array}{c}\text { Total } \\
\text { (n) }\end{array}$ & $\begin{array}{l}\text { Años de } \\
\text { estudio }\end{array}$ & $\begin{array}{r}\text { Benigno } \\
\text { (n) }(\%\end{array}$ & $\begin{array}{l}\text { Maligno } \\
\text { de todos }\end{array}$ & $\begin{array}{l}\text { Mayor } \\
\text { os tumore }\end{array}$ & Menor & AP & CAQ & CME \\
\hline Presente estudio & 286 & $2006-2016$ & $\begin{array}{c}230 \\
(80,42)\end{array}$ & $\begin{array}{c}56 \\
(19,58)\end{array}$ & $\begin{array}{c}274 \\
(95,8)\end{array}$ & $\begin{array}{c}12 \\
(4,2)\end{array}$ & $\begin{array}{c}172 \\
(60,14)\end{array}$ & $\begin{array}{c}9 \\
(3,14)\end{array}$ & $\begin{array}{c}14 \\
(4,9)\end{array}$ \\
\hline Inglaterra ${ }^{15}$ & 741 & 1974 - 2005 & $\begin{array}{c}481 \\
(64,9)\end{array}$ & $\begin{array}{c}260 \\
(35,1)\end{array}$ & $\begin{array}{c}281 \\
(38,2)\end{array}$ & $\begin{array}{c}455 \\
(61,8)\end{array}$ & $\begin{array}{c}329 \\
(44,4)\end{array}$ & $\begin{array}{c}62 \\
(8,4)\end{array}$ & $\begin{array}{c}85 \\
(11,5)\end{array}$ \\
\hline Brasil $^{10}$ & 599 & $1996-2005$ & $\begin{array}{c}469 \\
(78,3)\end{array}$ & $\begin{array}{c}130 \\
(21,7)\end{array}$ & $\begin{array}{c}512 \\
(86,5)\end{array}$ & $\begin{array}{c}87 \\
(14,5)\end{array}$ & $\begin{array}{c}406 \\
(67,8)\end{array}$ & $\begin{array}{c}39 \\
(6,5)\end{array}$ & $\begin{array}{l}30 \\
(5)\end{array}$ \\
\hline China $^{2}$ & 6.982 & $1985-2007$ & $\begin{array}{c}4.743 \\
(67,93)\end{array}$ & $\begin{array}{c}2.239 \\
(32,07)\end{array}$ & $\begin{array}{c}5.002 \\
(71,64)\end{array}$ & $\begin{array}{c}1.980 \\
(28,36)\end{array}$ & $\begin{array}{c}3.281 \\
(46,99)\end{array}$ & $\begin{array}{c}681 \\
(9,75)\end{array}$ & $\begin{array}{c}673 \\
(9,64)\end{array}$ \\
\hline India ${ }^{13}$ & 684 & $1991-2006$ & $\begin{array}{l}422 \\
(62)\end{array}$ & $\begin{array}{l}262 \\
(38)\end{array}$ & $\begin{array}{l}534 \\
(78)\end{array}$ & $\begin{array}{l}534 \\
(22)\end{array}$ & $\begin{array}{l}363 \\
(53)\end{array}$ & $\begin{array}{c}66 \\
(9,65)\end{array}$ & $\begin{array}{c}49 \\
(7,16)\end{array}$ \\
\hline Croacia $^{9}$ & 779 & $1985-2009$ & $\begin{array}{c}500 \\
(64,2)\end{array}$ & $\begin{array}{c}279 \\
(35,8)\end{array}$ & $\begin{array}{c}567 \\
(72,8)\end{array}$ & $\begin{array}{c}212 \\
(27,2)\end{array}$ & $\begin{array}{c}368 \\
(47,24)\end{array}$ & $\begin{array}{c}94 \\
(12)\end{array}$ & $\begin{array}{c}67 \\
(8,6)\end{array}$ \\
\hline
\end{tabular}

*AP: Adenoma Pleomorfo, CAQ: Carcinoma Adenoideo Quístico, CME: Carcinoma Mucoepidermoide.

tándose en $4,7 \%$ de todos los casos registrados, y en $25 \%$ de las neoplasias malignas, resultado similar a estudios en Croacia y Chile, ${ }^{6,9}$, sin embargo, la literatura muestra gran variación respecto a las frecuencias de las lesiones neoplásicas malignas de glándulas salivales sin presentar un patrón común.

En el presente estudio, las neoplasias epiteliales de glándulas salivales fueron más frecuentes en glándulas mayores, de manera similar a la literatura nacional e internacional (Chile ${ }^{4,6,7}$, $\mathrm{China}^{2}$, India $^{13}$, Brasil $^{10,14}$ y Croacia ${ }^{9}$ ) con excepción de Inglaterra en donde se reportó una frecuencia mayor en glándulas salivales menores ${ }^{15}$ (Tabla 4 ). Este predominio de los tumores de glándulas salivales mayores podía explicarse por el hecho de que el presente estudio se realizó en un centro de salud terciario que recibe a un gran número de pacientes derivados con tumores parotídeos. Es posible que los tumores de glándulas salivales menores sean diagnosticados y tratados en servicios de menor complejidad, mientras que solo las neoplasias de glándulas salivales mayores se envían a nuestro servicio hospitalario.

De acuerdo a la localización, las neoplasias de glándulas salivales fueron más frecuentes en glándula parótida $(82,8 \%)$, seguido por la glándula submandibular $(12,9 \%)$ y luego por las glándulas salivales menores $(4,2 \%)$, lo cual es similar a varios resultados reportados en la literatura ${ }^{6,10}$. Sin em- bargo, hay algunos estudios que presentaron más casos en paladar que en glándula submandibular, por lo tanto, aún no existe un patrón definido ${ }^{2,12,14}$. No registramos ningún caso en glándula sublingual de manera similar a otros estudios ${ }^{10,12,14}$, lo cual confirma la rareza de tumores salivales en esta zona.

En relación a la distribución por género, pudimos observar que los tumores de glándulas salivales son más frecuentes en mujeres, con una proporción hombre-mujer de 1:1,86. Estos resultados son similares a los registros nacionales $^{4,6}$, pero distan de los datos registrados a nivel mundial, en donde la proporción de neoplasias de glándulas salivales es mayor para mujeres pero menos marcada $(0,9: 1-1,1: 1)^{2,14,15}$.

La sexta década de vida (51 a 60 años) fue el rango etario con más casos registrados para las neoplasias benignas $(21,3 \%)$, mientras que la cuarta década fue la más frecuente para casos de neoplasias malignas (19,6\%). Los promedios de edad para neoplasias benignas y malignas fueron de $47( \pm 17)$ y $52( \pm 18)$ años respectivamente. Estos resultados son bastante similares a los registros nacionales previos, en donde se ha informado que la edad media para neoplasias benignas es de 45 años y neoplasias malignas es de 52 años, y que el período entre la cuarta y sexta década de vida son los períodos donde más se registran $\operatorname{casos}^{4,6}$. 


\section{Conclusión}

Las neoplasias de glándulas salivales son un grupo de patologías poco comunes que represen$\tan 3$ a $6 \%$ de los tumores de cabeza y cuello, y $0,6 \%$ de todas las neoplasias. Sin embargo, a pesar de su baja frecuencia presentan una gran variedad de tipos histológicos con comportamientos clínicos y manejo terapéutico totalmente distintos.

La evidencia disponible en la actualidad varía mucho de un país a otro, y a nivel nacional no hay suficientes registros actualizados que permitan tener una idea de la frecuencia y presentación de los distintos tipos histológicos de las neoplasias de glándulas salivales.

Los resultados han mostrado ser similares a otros registros nacionales, sin embargo, aún se requieren más estudios para poder establecer un análisis más representativo y actualizado.

\section{Referencias}

1. Shah JP, Patel SG, Singh B, Shah JP. Jatin Shah's head and neck surgery and oncology Philadelphia: Elsevier/ Mosby; 2012.

2. Tian Z, Li L, Wang L, Hu Y, Li J. Salivary gland neoplasms in oral and maxillofacial regions: a 23-year retrospective study of 6982 cases in an eastern Chinese population. Int J Oral Maxillofac Surg 2010; 39 (3): 23542.

3. Neville BW, Damm DD, Chi AC, Allen CM. Oral and Maxillofacial Pathology: Elsevier; 2015.

4. Larraín D, Madrid MA, Capdeville F, Ferrada VC. Tumores de glándulas salivales: hallazgos histopatológicos en 168 pacientes. Rev Chilena Cir 2005; 57 (5): 373-8.
5. Jaber MA. Intraoral minor salivary gland tumors: a review of 75 cases in a Libyan population. Int J Oral Maxillofac Surg 2006; 35 (2): 150-4.

6. Celedón LC, Ojeda ZJP, Agurto VM, Olavarría LC, Paredes WA, Niklischek BE. Tumores de glándulas salivales. Experiencia de 20 años. Rev Otor Cir Cabeza y Cuello 2002; 62: 255-64.

7. Ortega JT. Tumores de las glándulas salivales. Rev Chilena Cir 1999; 51 (4): 334-40.

8. Barnes L. Pathology and genetics of head and neck tumours: IARC; 2005.

9. Luksic I, Virag M, Manojlovic S, Macan D. Salivary gland tumours: 25 years of experience from a single institution in Croatia. J Craniomaxillofac Surg 2012; 40 (3): e75-81.

10. de Oliveira FA, Duarte EC, Taveira CT, Maximo AA, de Aquino EC, Alencar Rde C, et al. Salivary gland tumor: a review of 599 cases in a Brazilian population. Head Neck Pathol 2009; 3 (4): 271-5.

11. Ito FA, Ito K, Vargas PA, de Almeida OP, Lopes MA. Salivary gland tumors in a Brazilian population: a retrospective study of 496 cases. Int J Oral Maxillofac Surg 2005; 34 (5): 533-6.

12. Al-Khateeb TH, Ababneh KT. Salivary tumors in north Jordanians: a descriptive study. Oral Surg Oral Med Oral Pathol Oral Radiol Endod 2007; 103 (5): e53-9.

13. Subhashraj K. Salivary gland tumors: a single institution experience in India. Br J Oral Maxillofac Surg 2008; 46 (8): 635-8.

14. Vasconcelos AC, Nor F, Meurer L, Salvadori G, Souza LB, Vargas PA, et al. Clinicopathological analysis of salivary gland tumors over a 15 -year period. Braz Oral Res 2016; 30.

15. Jones AV, Craig GT, Speight PM, Franklin CD. The range and demographics of salivary gland tumours diagnosed in a UK population. Oral Oncology 2008; 44 (4): 407-17. 